\title{
A Screening Study of Corn Oil versus Fish Oil and Coconut Oil on Biochemical Cardiovascular Risk Factors in Rats
}

\author{
Fawzy M. Lashin ${ }^{1}$ and Hanan A. Rizk ${ }^{2}$, Omar A. Ahmed-Farid ${ }^{3}$ and Ahmed M. \\ Shehata $^{4}$ \\ ${ }^{1}$ Hormone Evaluation Dept., ${ }^{2}$ Pharmacology Department, ${ }^{3,4}$ Physiology Department, National \\ Organization for Drug Control and Research (NODCAR), Giza 12553, Egypt. \\ Received: 10 May 2020 / Accepted 30 June 2020 / Publication date: 20 July 2020
}

\begin{abstract}
This study aimed to investigate the screening effect of edible oil consumption on biochemical cardiovascular risk factors in male albino rats. Twenty four rats $200 \pm 25 \mathrm{~g}$ were divided into four groups. The first group fed on basal diet and considered as control. The second, third and fourth group were fed on basal diet and taken separately orally $0.4 \mathrm{ml} / 100 \mathrm{~g}$ b.w /day from corn oil, fish oil and coconut oil for 60 days. At the end of experimentally, lipid profile (T.Ch, TG, HDL, LDL and Total Phospholipids), liver function (ALT, AST, TP, Alb and Glob), oxidative stress markers (MDA, GSH, and NO) were determined. Glucose level, cell energy (ATP), appetite marker, serotonin (5HT) was measured as well as Aortic tissue histopathological examination. Results showed negative alteration in lipid profile, liver function, oxidative stress markers and histopathological examination of aortic tissue for corn oil administered group as compared with the control group. In contrast fish, oil and coconut oil showed normal physiological response for all parameters except pathohistological feature in coconut oil group. Data concluded that the corn oil oral administration triggered risk factors for cardiovascular disease. While fish oil and coconut oil administration showed protective activity against these factors with the superiority of fish oil over coconut oil
\end{abstract}

Keywords: Corn oil, fish oil, coconut oil, lipid profile, liver function, ATP, serotonin.

\section{Introduction}

Cardiovascular diseases (CVDs) represent a general category of diseases affecting the cardiovascular system, which includes both coronary heart disease (CHD) and heart failure (Masters et al., 2013). The main reason for the decreasing in blood flow may be due to the thickening and hardening of the arteries that nourish the heart muscle (Leong et al., 2008). Congestive heart failure is a disorder in which the heart loses its ability to pump blood efficiently, resulting in stroke or stroke due to an explosion of blood vessels that transport oxygen and nutrients to the brain (He et al., 2001). A high blood concentration of total cholesterol, triacylglycerol, and LDL cholesterol and a low blood concentration of HDL cholesterol are considered important risk factors for the development of CVDs and have been documented for many years (Kannel et al., 1979; O'Donnell and Elosua, 2008 and Hajar, 2017). Hypercholesterolemia and its associated CVDs previously discussed, studies have shown that lowering cholesterol reduces the risk of coronary heart disease and reduces mortality (Plat and Mensink, 2000).

The type of fat in the diet has since long been associated with long-term health outcomes, mainly regarding blood lipids and CDV. Hence, Dietary fat selection is known to exert a major influence on circulating cholesterol levels; they are raised with the consumption of fats containing saturated fatty acids (SFA), and reducing fats rich in monounsaturated (MUFA) and polyunsaturated (PUFA) fatty acids (Mensink, and Katan, 1992). It is well known that the intake of saturated fatty acids (SFA) increases blood triacylglycerides (TG), total cholesterol (TC), low-density lipoprotein cholesterol (LDL) and decreases high-density lipoprotein cholesterol (HDL). That leading to a higher risk for CVD and studies recommended the need to reduce the level of diet in SFA (Gylling and Meittinen 1999; Hallikainen et al., 2000; Mason et al., 2009 and Yamagishi et al., 2010).

Corn oil is a by-product of corn meal- and starch-making companies and is mainly used in fastfood restaurants. Fatty acid composition of corn oil comprises a relatively high level of linoleic acid (58-62\%) as the major n-6 PUFA essential fatty acid (Eid et al., 2019). Refined corn oil is composed

Corresponding Author: Fawzy m. Lashin, National Organization for Drug Control and Research (NODCAR), Giza 12553, Egypt. E-mail: Fawzy870@hotmail.com 
of PUFA 59\%, MUFA $24 \%$, and SFA $13 \%$ with a ratio of linoleic acid (LA) to n3 fatty acid alphalinolenic acid (ALA) LA/ALA of 83. Corn oil contains also gamma- and alpha-tocopherols and betasitosterol (Savva and Kafatos, 2015). The percentages of ALA (C18:3) lower than 1.5\%, therefore, corn oil cannot be considered a source of omega- 3 fatty acids in the diet. While the second major fatty acid is oleic (Barrera-Arellano et al., 2018). Another study results by Carrillo et al, (2017) that were in accordance with values reported by other authors. The study recorded that fatty acids of corn oil were: $\mathrm{C} 16: 0$ palmitic acid with $12.57 \%$ of fatty acids content, C18:0 stearic acid with $2.02 \%$ of fatty acids content, C18:1 oleic acid with $29.70 \%$ of fatty acids content, C18:1 trans elaidic acid with $0.81 \%$ of fatty acids content, C18:2 linoleic acid with $52.68 \%$ of fatty acids content, $\mathrm{C} 18: 3$ linolenic acid with $1.12 \%$ of fatty acids content, and finally $\mathrm{C} 20: 0$ arachidic acid with $0.27 \%$ of fatty acids content.

Coconut oil is one of edible oils obtained from the extraction of coconut kernel. The virgin coconut oil has emerged as functional food oil due to its capability to provide some beneficial biological activities to human health due to its retained some minor components like tocopherols and phenolics compounds (Rohman et al., 2019). In addition, because of the high-saturated fatty acid and fat contents, coconut oil is resistant to oxidative modifications, which make them suitable for cooking (Narayanankutty et al., 2018). The predominant fatty acids of the virgin coconut oil were the medium chain fatty acids lauric acid (C12:0) (31-47 percent) followed by myristic acid (C14:0) (18-25 percent), the concentrations of which increased with the kernel maturity. The concentrations of the small chain saturated fattyacids, namely, caprylic acid (C8:0), ranging between 1 to 7.5 percent, and capric acid $(\mathrm{C} 10: 0)$, ranging between 2 to 7.7 percent, with the increasing maturity of the nut. The long chain fatty acids, palmitic $(\mathrm{C} 16: 0)$ and stearic $(\mathrm{C} 18: 0)$ acids ranged between 7 to 24 percent of the total fatty acids, while the unsaturated fatty acids oleic (C18:1), linoleic (C18:2) and linolenic (C18:3) acids together made up only 2 to 6 percent. C16:0 and C18:0 were lesser in the more mature nuts, while the unsaturated fatty acids increased marginally with nut maturity, but the concentrations were on par among the 10 and 12-month-old kernel (Azeez, 2007).

Fish oil is a common term used to refer to two kinds of omega-3 fatty acids: eicosapentaenoic acid (EPA) and docosahexaenoic acid (DHA). These omega-3 fats are usually found in fish, animal products, and phytoplankton. The fatty acids EPA and DHA are involved in regulating various biological processes such as the inflammatory response, various metabolic signaling pathways, and brain function (Burhani and Rasenick, 2017). They can be synthesized in the body from alphalinolenic acid (ALA), but in small amounts for most people. Fish oil, which is thought to be favorable for dyslipidemia and immune disorders, contains high levels of n-3 polyunsaturated fatty acids (PUFAs), such as EPA and DHA (Gomaa et al., 2016 and Wooten et al., 2016).

The present study was designed to investigate the effect of corn oil, coconut oil or fish oil oral supplementation on some biochemical parameters and histological investigation of arterial sections of thoracic aorta of normal male rats.

\section{Materials and Methods}

\section{Animals and oils}

In this experimental study, twenty adult male Sprague-Dawley rats (weight about $200 \pm 25 \mathrm{~g}$ ) were selected from the laboratory animal house of NODCAR. Rats were maintained under standard conditions (12 hr dark/night. Animals were allowed to feed free on AIN-93G diet in pelleted form for complete diet composition as a standard diet (SD) and free for tap water throughout the experiment (Reeves et al., 1993). The food debris, faeces and urine were removed daily to prevent food and water contamination. Corn, coconut and fish oils were purchased from a local market, Cairo, Egypt. All animal experimentation protocols were carried out under the supervision of the Ethics Committee of the national organization of drug control and research (NODCAR), Egypt.

\section{Experiment design}

Rats were randomly divided into four different experimental groups: The 1st control left without any treatment. The 2 nd group treated with $0.4 \mathrm{ml} / 100 \mathrm{~g} \mathrm{~b} . \mathrm{w}$ corn oil. The $3 \mathrm{rd}$ group treated with fish oil $0.4 \mathrm{ml} / 100 \mathrm{~g}$ b.w and the $4^{\text {th }}$ group treated with coconut oil $0.4 \mathrm{ml} / 100 \mathrm{~g}$ b.w. treatments were orally using oral gavage and extended daily for 60 days. At the end of the treatment period, the 
rats were anaesthetized using diethyl ether, and blood samples were collected from Retro-orbital plexus, centrifuged immediately after collection, and serum then stored at $-20^{\circ} \mathrm{C}$ for biochemical assays.

\section{Biochemical analyses:}

Serum used for the determination of the lipid parameters calorimetrically, total cholesterol (TC), triglycerides (TG), high-density lipoprotein cholesterol (HDL) and low-density lipoprotein cholesterol (LDL) using Stanbio Liqui Color ${ }^{\circledR}$ Kits, (Proc. Nos. 1010; 2100; 0590 and 0710, respectively), prosuced by Stanbio Laboratory Inc., Boerne, Texas, USA. While the total phospholipids determined according to Baginski et al, (1972). In addition to transaminases, aspartate aminotransferase (ASAT) and alanine aminotransferase (ALAT) calorimetrically and proteins, total protein (TP), albumin (Alb), globulin (Glob) (calculated as Glob = TP-Alb) and glucose (Glu) using kits from Quimica Clinica Aplicada S.A. (Amposta, Spain). All procedures and calculations were as described by the manufacturer's manuals. Serum tissue also used for the determination of malondialdehyde (MDA), nitric oxide (NO), glutathione (GSH) and adenosine triphosphate (ATP) by HPLC according to Karatep, (2004), Papadoyannis et al, (1999), Jayatilleke and Shaw, (1993) and Teerlink et al, (1993), respectively.

\section{Brain tissue preparation}

Each brain tissue was homogenized in $75 \%$ aqueous HPLC grade methanol $(10 \% \mathrm{w} / \mathrm{v})$. The homogenate was spun at 4000 r.p.m. for 15 minutes and the supernatant was isolated and used for serotonin or5-hydroxytryptamine (5-HT) assay by HPLC according to Pagel et al. (2000).

\section{Histopathological examination:}

Arterial sections of thoracic aorta Aorta samples were taken from different groups and fixed in $10 \%$ neutral buffered formalin for twenty-four hours. Tissues were dehydrated using serial dilutions of ethyl alcohol (70, 80, 90, 95 and 100\%). washed by water then were used for dehydration in serial dilutions of alcohol (methyl, ethyl and absolute ethyl). The samples were cleared in xylene and embedded in paraffin for $24 \mathrm{~h}$ at $560 \mathrm{C}$. The paraffin wax tissue blocks were sectioned into $4-\mathrm{mm}$ slices using a microtome. Then, the tissue sections were deparaffinized and stained with hematoxylin and eosin (H\&E). The histopathological examinations were conducted using a light microscope (Banchroft et al., 1996).

\section{Statistical analysis}

The values were expressed as the mean $\pm \mathrm{SE}$ for the 6 rats in each group. Differences between groups were assessed by one-way analysis of variance (ANOVA) using SAS. Statistical analysis of the obtained data was performed using the general linear model (GLM). Significant differences among means were evaluated using Duncan's Multiple Range Test.

\section{Results}

Results about the biochemical assays represented in Table (1). Data showed the alteration of lipid profile as compared to the control values, the corn oil group recorded significant increase in TC, LDL levels and significant decreases in HDL and total Phospholipids (T. Ph) levels $(\mathrm{p}<0.05)$. While, the coconut oil and fish oil groups showed significant increases in $\mathrm{T}$. $\mathrm{Ph}(\mathrm{p}<0.05)$.The coconut oil and fish oil groups recorded significant increase in HDL and T. Ph levels and significant decreases in LDL level as compared to the corn oil group corresponding values.

Transaminases, ALT and AST enzymes activities elevated significantly in corn oil group as compared to the control values. Meanwhile, the aforementioned enzymes activities decreased significantly in both coconut oil and fish oil groups as compared to the corn oil group corresponding values.

Albumin (Alb) level decreased significantly in corn oil group as compared to the control value. While, all measured proteins not statistically different in coconut oil and fish oil groups as compared to the corn oil group corresponding values.

As regard to the oxidative stress related parameters, MDA and NO levels increased significantly 
and GSH decreased significantly in the corn oil group as compared to the control values. The coconut oil and fish oil groups recorded significant decrease in MDA and NO levels and significant increases in GSH level as compared to the corn oil group corresponding values at $(p<0.05)$. In the meantime, results of the fish oil group showed advantage over the coconut oil through the significant decrease and increase in the MDA and GSH levels, respectively as compared with the coconut oil group corresponding values.

The corn oil group showed significant decrease in ATP level as compared to the control level. The coconut oil group recorded significant increase in 5HT level as compared to the control and corn oil groups levels, and ATP level significantly increasedas compared to the corn oil group level but significantly decreased as compared to the Fish oil group level at $(\mathrm{p}<0.05)$. The fish oil group recorded significant increase in ATP and 5HT levels as compared to the control and corn oil groups corresponding levels at $(\mathrm{p}<0.05)$.

\section{The histopathological results}

Aortic histophatological examination of the experimental groups revealed normal tunica intimae, tunica media and tunica adventitia in the control group (Figure 1). In corn oil group irregular tunica intimae with more foam cells and irregular tunica media with dense irregular nuclei showed in Figure (2). The coconut oil group showed milled irregular tunica intimae with slight foam cells and irregular tunica media and dense irregular nuclei Figure (3). While, fish oil group showed normal tunica intimae, tunica media and tunica adventitia Figure (4).

Table 1: Effect of each orally administered corn, fish and coconut oils on serum biochemical parameters

\begin{tabular}{|c|c|c|c|c|}
\hline \multicolumn{3}{|l|}{ Item } & Fish Oil & Coconut Oil \\
\hline \multicolumn{5}{|l|}{ Lipid profile } \\
\hline $\mathrm{TC} \mathrm{mg} / \mathrm{dl}$ & $53.60 \pm 1.77$ & $60.40 \pm 1.72^{\mathrm{a}}$ & $52.90 \pm 1.86^{\mathrm{b}}$ & $55.50 \pm 1.50$ \\
\hline TG mg/dl & $52.80 \pm 1.45$ & $53.30 \pm 2.09$ & $49.40 \pm 1.30$ & $55.20 \pm 1.75$ \\
\hline HDL mg/dl & $17.70 \pm 1.10$ & $14.70 \pm 0.59^{\mathrm{a}}$ & $18.10 \pm 0.83^{b}$ & $17.90 \pm 0.76^{b}$ \\
\hline LDL mg/dl & $25.30 \pm 1.69$ & $35.10 \pm 2.19^{\mathrm{a}}$ & $25.00 \pm 1.79^{b}$ & $26.60 \pm 1.94^{\mathrm{b}}$ \\
\hline T. $\mathrm{Ph} \mathrm{mg} / \mathrm{dl}$ & $55.30 \pm 2.19$ & $37.20 \pm 2.10^{\mathrm{a}}$ & $65.8 \pm 3.14^{\mathrm{ab}}$ & $63.20 \pm 3.43^{\mathrm{ab}}$ \\
\hline \multicolumn{5}{|l|}{ Transaminases } \\
\hline ALT U/L & $40.60 \pm 1.18$ & $52.00 \pm 1.96^{\mathrm{a}}$ & $37.10 \pm 1.99^{b}$ & $34.00 \pm 1.46^{\mathrm{b}}$ \\
\hline AST U/L & $64.90 \pm 2.62$ & $87.40 \pm 1.72^{\mathrm{a}}$ & $63.20 \pm 1.44^{\mathrm{b}}$ & $67.90 \pm 3.28^{\mathrm{b}}$ \\
\hline \multicolumn{5}{|l|}{ Protein } \\
\hline TP g/dl & $6.80 \pm 0.17$ & $6.40 \pm 0.08$ & $6.80 \pm 0.17$ & $6.90 \pm 0.13$ \\
\hline Alb g/dl & $4.20 \pm 0.09$ & $3.90 \pm 0.06^{\mathrm{a}}$ & $4.10 \pm 0.06$ & $4.20 \pm 0.08$ \\
\hline Glob g/dl & $2.60 \pm 0.16$ & $2.50 \pm 0.09$ & $2.70 \pm 0.17$ & $2.70 \pm 0.15$ \\
\hline \multicolumn{5}{|c|}{ Oxidative stress related items } \\
\hline MDA nmol/dl & $28.0 \pm 0.93$ & $44.40 \pm 2.15^{\mathrm{a}}$ & $29.50 \pm 1.23^{\mathrm{b}}$ & $36.70 \pm 1.36^{\mathrm{abc}}$ \\
\hline $\mathrm{GSH} \mu \mathrm{mol} / \mathrm{dl}$ & $18.80 \pm 0.91$ & $12.70 \pm 1.02^{\mathrm{a}}$ & $23.80 \pm 1.78^{b}$ & $17.20 \pm 1.44^{\mathrm{bc}}$ \\
\hline $\mathrm{NO} \mu \mathrm{mol} / \mathrm{dl}$ & $0.64 \pm 0.04$ & $1.00 \pm 0.09^{\mathrm{a}}$ & $0.53 \pm 0.05^{\mathrm{b}}$ & $0.66 \pm 0.06^{\mathrm{b}}$ \\
\hline \multicolumn{5}{|c|}{ Energy homeostasis related items } \\
\hline ATP $\mu \mathrm{g} / \mathrm{dl}$ & $22.50 \pm 1.33$ & $16.90 \pm 0.70^{\mathrm{a}}$ & $27.80 \pm 1.7^{\mathrm{ab}}$ & $20.80 \pm 1.34^{\mathrm{bc}}$ \\
\hline Glu g/dl & $104.90 \pm 5.11$ & $129.60 \pm 10.65$ & $120.50 \pm 6.98$ & $105.60 \pm 13.45$ \\
\hline $5 \mathrm{HT} \mu \mathrm{g} / \mathrm{g}$ & $0.61 \pm 0.03$ & $0.64 \pm 0.15$ & $0.75 \pm 0.06^{\mathrm{ab}}$ & $0.73 \pm 0.13^{\mathrm{ab}}$ \\
\hline
\end{tabular}

Data are expressed as Mean \pm S.E.M for 6 rats /group. Superscripts indicate significance $\mathrm{P}<0.05$, (a) indicates significance from control group, (b) indicates significance from corn oil group and (c) indicates significance from fish oil group. 


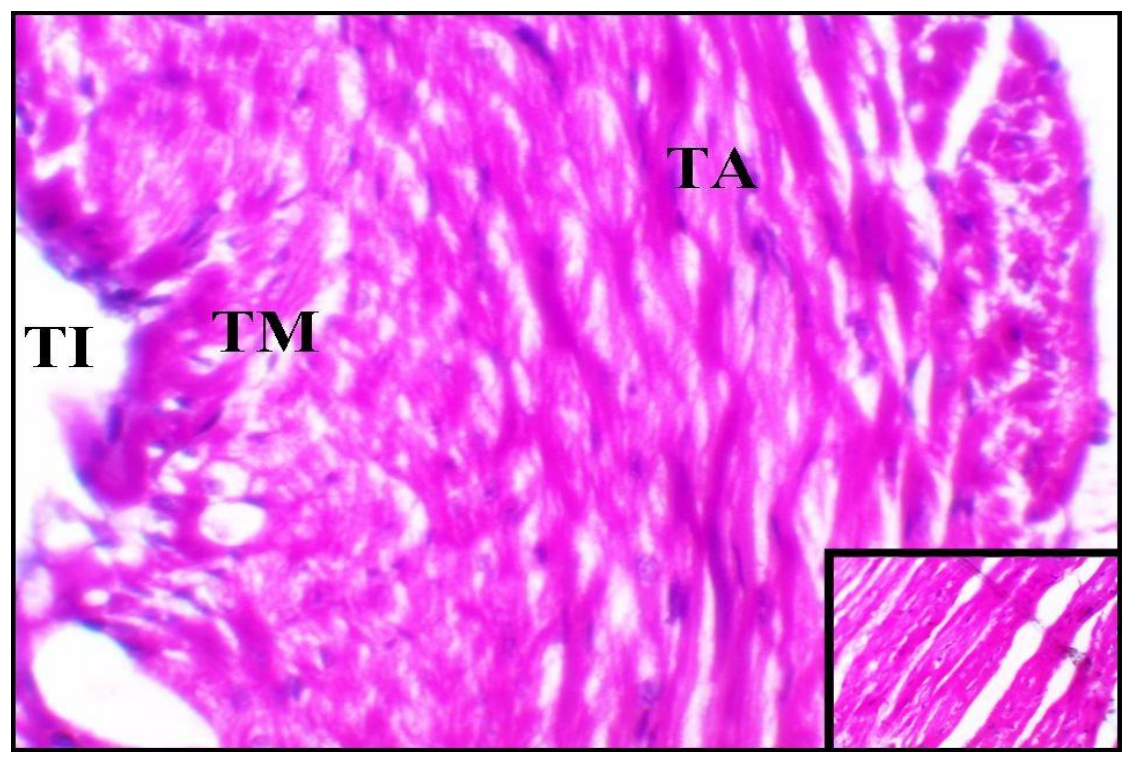

Fig. 1: Aortic histophatological image of control group showed normal tunica intimae (TI), tunica media (TM), and tunica adventitia (TA) H\&E 400.

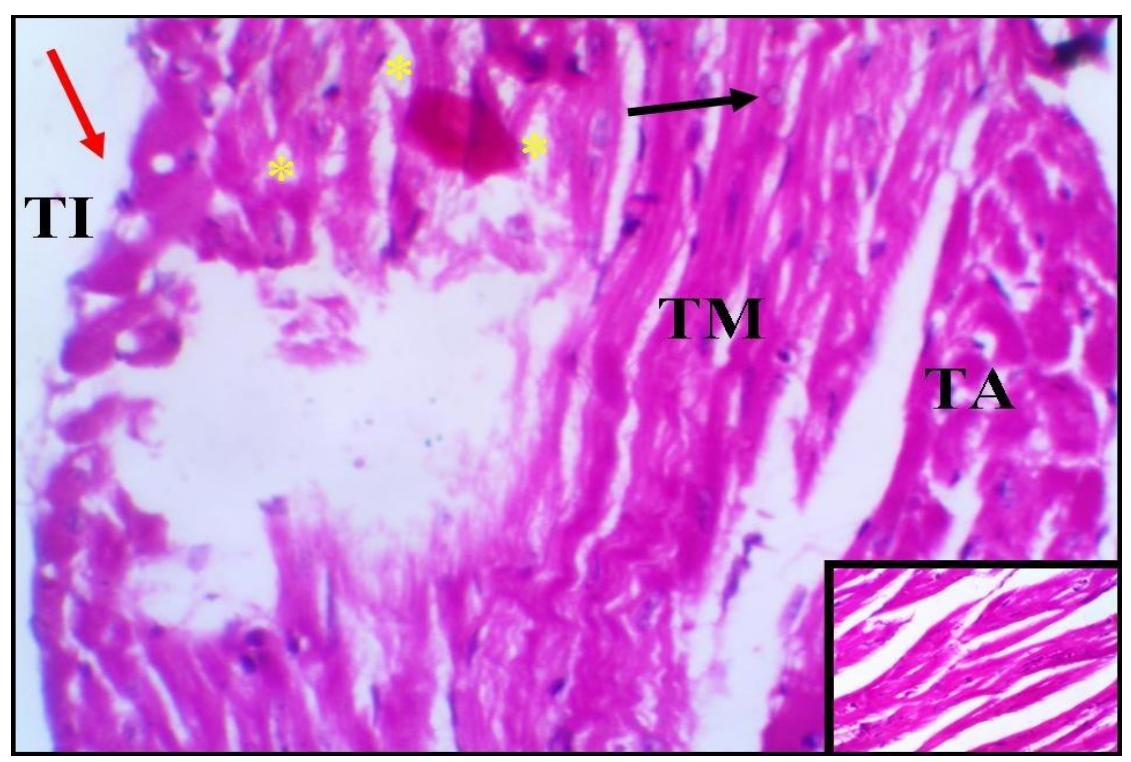

Fig. 2: Aortic histophatological image of corn oil group showed irregular tunica intimae (TI) red arrows with more foam cells yellow stars, irregular tunica media (TM), and dense irregular nuclei (black arrows) H\&E 400. 


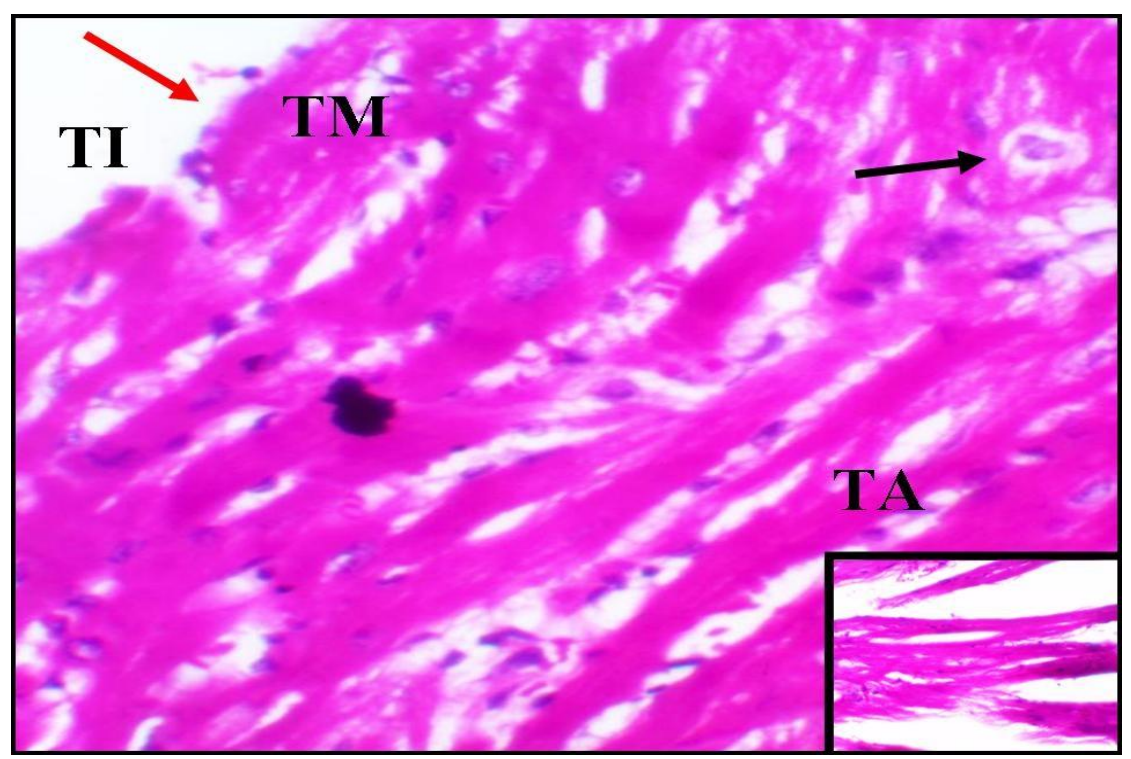

Fig. 3: Aortic histophatological image of fish oil group showed normal tunica intimae (TI), tunica media (TM), and tunica adventitia (TA) H\&E 400.

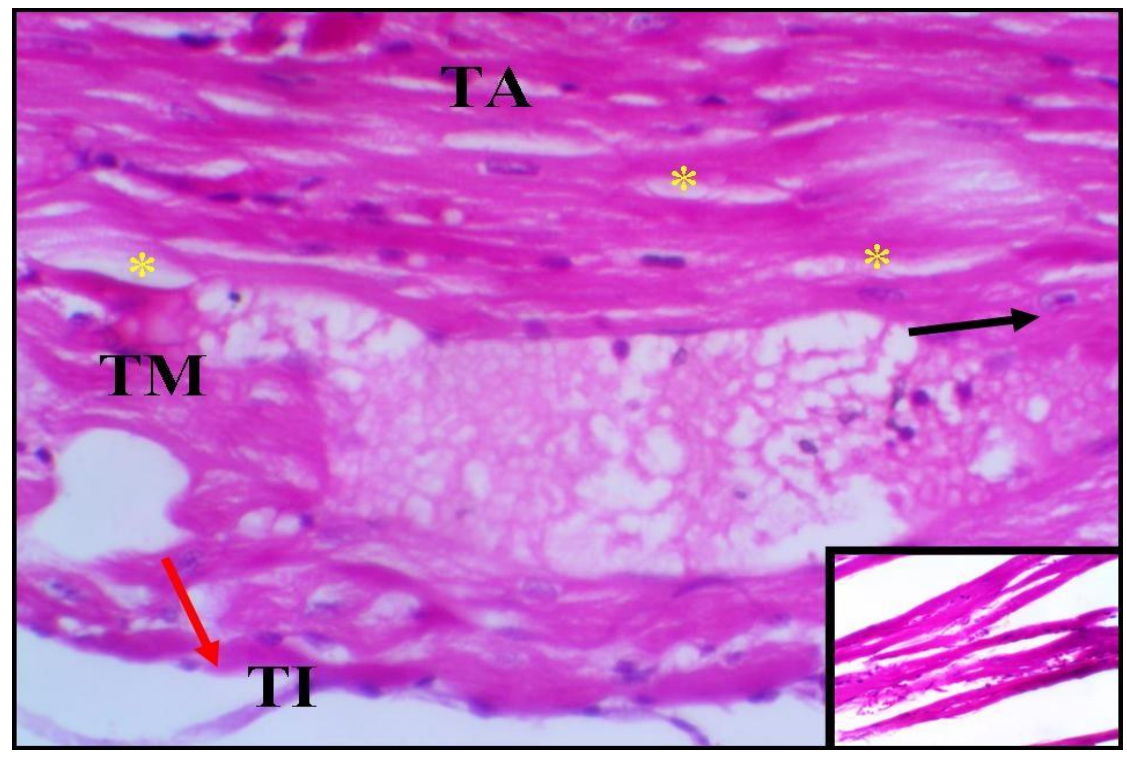

Fig. 4: Aortic histophatological image of coconut oil group showed milled irregular tunica intimae (TI) red arrows with slight foam cells yellow stars, irregular tunica media (TM), and dense irregular nuclei (black arrows) H\&E 400.

\section{Discussion}

In the present study, all supplemented oils are physically and chemically different from each other and have different fatty acid profiles as previously mentioned that may functionally lead to the recorded effects on the measured markers. Corn oil supplementation showed as compared to the standard diet-fed normal control group, a significant increase in TC, LDL, MDA, NO levels and ALT and AST enzymes activities and significant decreases in HDL, T.Ph, Alb, GSH and ATP levels. In line with irregular tunica intimae with foam cells and irregular tunica media with dense irregular, nuclei were recorded through the aortic histopathological examination. Corn oil feeding previously caused significant increases in liver and blood MDA levels in rats as compared with olive and sunflower oils. Concluding that oils rich in polyunsaturated fatty acids (PUFA) increases lipid peroxidation significantly and may raise the susceptibility of tissues to free radical oxidative damage 
(El-Sayed et al., 2014).

Corn oil contains a high level of linoleic acid (LA) and low level of alpha-linolenic acid (ALA) and oleic acid that may be related to the predicted effects by its oral supplementation in rats. High intake of linoleic acid increases the linoleic acid content of very-low-density lipoprotein (VLDL) and HDL that leads to increase their susceptibility to oxidize that increases the risk of CVD (Reaven et al., 1993; Francis, 2000; Young et al., 2003 and Shao et al., 2006). Atherosclerosis development started by LDL oxidation which caused direct toxic effects to the cell, recruitment and entry of monocytes into the subendothelial layer and increased foam cell formation in the blood vessel walls (Foam cells are a type of cell that contain cholesterol) (Gumbiner et al., 1998), leading to increased atherosclerosis and inflammation (Silaste et al., 2004). In addition consuming more linoleic acid increases the amount of linoleic acid in complicated aortic plaques (Dayton et al., 1965). Oxidized linoleic acid metabolites can then induce direct toxic effects to the endothelium such as inflammation, reactive oxygen species and adhesion molecules causing endothelial activation and permeability and a greater number of lipoproteins entering into the subendothelium and reduced aortic relaxation leading to atherosclerosis (Nicolosi et al., 2002; Eiselein et al., 2007 and Wang et al., 2009). The Food and Drug Administration (FDA) supports the claim that oleic acid reduces the risk of CVD. Oils had contained up to $60 \%$ oleic acid, which may make it a product that is beneficial for vascular health. Hence, high oleic acid levels will be able to claim that they may reduce the risk of coronary heart disease (Voelker, 2019).

As regard to oxidative stress, MDA, an oxidation product of both linoleic acid and arachidonic acid, is generally used as an indicator of lipid peroxidation numerous studies have found increased lipid peroxidation products, measured via thiobarbituric acid reactive substances, in patients with atherosclerosis (Jira et al., 1998). Hypercholesterolemia stimulates polymorphonuclear leukocytes and activates vascular endothelial cells, which results in excess reactive oxygen species (ROS) release (Prasad and Kalra, 1993 and Prasad, 1997).

NO is synthesized by endothelial cells from L-arginine and oxygen and the endothelial nitric oxide synthase synthesize NO which is calcium-dependent. The enzyme is responsible for NO production in healthy blood vessels. Elevated oxidative stress causes the production of peroxynitrites. NO production induces Guanylate cyclase to the synthesis of cGMP from cGTP. The last molecule causes cellular hyperpolarization due to the activation of the potassium canals. These reactions cause the inhibition of the entrance of calcium and, in this way, the vasodilatation in the cardiovascular system. Endothelial dysfunction is the reduction in NO availability. Increase in oxidative stress with NO degradation and Decrease in NOs synthesis (effect of oxidized-LDL). NO, oppose the atherogenic stimuli preventing vascular structural modifications (Raddino et al., 2007). A healthy endothelium with the normal generation of $\mathrm{NO}$ acts to prevent atherosclerosis development and its complications. In contrast to the beneficial effects of $\mathrm{NO}$ at physiologic concentrations, large amounts of $\mathrm{NO}$ can be toxic and proinflammatory (Bian et al., 2008).

Glutathione (GSH), a sulfhydryl (SH)-containing tripeptide, has several major physiological functions: it maintains SH groups of proteins in a reduced state, participates in amino acid transport, detoxifies foreign compounds, enzymatically degenerates endogenous peroxides, forms bioactive molecules, and acts as a coenzyme in several enzymatic reactions. GSH has also been demonstrated to play a role in detoxifying oxygen radicals and therefore may prevent cellular damage from oxidative stress (Stamler and Slivka, 1996). There is evidence that orally administered GSH increases its plasma concentrations in animals and humans (Hagen and Jones, 1989). A previous study concluded that In conclusion, a reduced level of GSH may be an important risk factor for the development of CVD, which anticipated that oral administration of GSH is a possible therapeutic strategy for the prevention of CVD (Shimizu et al., 2004).

Serotonin (5HT) is synthesized in the central nervous system (CNS), where it modulates a variety of behavioral functions, including the regulation of sleep or wakefulness, appetite, nociception, mood, stress and maternal or sexual behavior (Jacobs and Azmitia, 1992). Serotonin also participates in numerous other physiological and homeostatic processes, such as gastrointestinal peristalsis, blood coagulation and the maintenance of blood pressure (Vanhoutte, 1987; Gershon, 1999 and Awabdy et al., 2003). Serotonin regulates vascular tone through the contraction and relaxation of blood vessels (Yildiz et al., 1998 and O'Connor et al., 2001). Serotonin effects are mediated by 5HT1B, 5-HT2A, 5-HT2B, 5-HT4 and 5-HT7 receptors, which are located on vascular smooth muscle cells and endothelial cells. The 5-HT2B receptor mediates either contraction or relaxation in the aorta 
and mesenteric arteries (Banes and Watts, 2003). A lack of serotonin signaling through the 5-HT2B receptors alters cardiomyocytecytoarchitecture and affects heart contractility (Nebigil et al., 2001). Hence, the level of circulating serotonin is a key factor in maintaining normal cardiovascular activity (Côté et al., 2004).

Drugs elevating 5-HT (specific serotonin reuptake inhibitors, SSRIs), they are suggested to increase 5-HT not only in brain synapses but also in cardiac tissue and therefore they might be implicated in arrhythmias which is an irregular heartbeat (Castro et al., 2013). Previous study examined therapeutic effects of anti-depression drug paroxetine and anti-anxiety drug buspirone that could improve the brain serotonin function in mice. Both drugs reduced cardiac enlargement and improved systolic dysfunction and symptoms of severe congestive heart failure in dilated cardiomyopathy mice (Li et al., 2012).

In the present study, coconut oil and fish oil supplementation significantly increased the serum total phospholipids and the brain serotonin levels and showed no alterations of the liver function enzymes, the serum proteins as compared to control group. While, as compared to the corn oil supplementation results, coconut oil and fish oil supplementation showed advantage by their significant increase in the lipid parameters HDL and T.Ph levels and significant decreases in LDL, MDA and NO levels as well as the ALT and AST enzymes activities. Significant increased in serum GSH and brain serotonin levels. Fish oil supplementation only showed the advantage over the other oils through significant decrease in TC as compared with the recorded in corn oil group level and the significant decrease in MDA and significant increase in GSH and ATP as compared to their correspondings in coconut oil group. That's in line with the normal aortic histological feature.

Several pharmacological effects have been reported in virgin coconut oil which include antioxidants, anti-inflammation, anti-hyperlipidemia and antibacterial activities (Narayanankutty et al., 2018 and Rohman et al., 2019). Coconut oil contains medium chain triglyceride that was reported to have beneficial effects on lipid metabolism due to the presence of lauric acid (Resende et al., 2016 and Narayanankutty et al., 2018). In contrast to the results of a recent study which concluded that coconut oil intake does not improve CVD biomarkers to support any clinical recommendation when compared to other vegetable oils like olive oil. Because coconut oil consumption may increase HDL, LDL and TC similarly to other, rich food sources of SFA (Santos et al., 2019).

Fish oil supplements have shown a hypotriglyceridemic effect, and a reduction in LDL levels when saturated fat intake is partially replaced by fish oil (Harris, 1989). A prospective cohort studies and secondary prevention trials have provided strong evidence that an increasing intake of n-3 fatty acids from fish or plant sources substantially lowers risk of cardiovascular mortality (Hu et al., 2001). Dietary n-3 long chain polyunsaturated fatty acids (n-3 LCPUFA), eicosapentaenoic acid (EPA), and docosahexaenoic acid (DHA) to cardioprotection (Demaison and Moreau, 2002 and Kris-Etherton et al., 2004). Consequently, increased consumption of food-based sources of EPA and DHA has been recommended in most nutritional guidelines issued by government and health organizations, such as American Heart Association (Lichtenstein et al., 2006).

Studies indicate that the use of fish oil is associated with coronary heart disease risk reduction. A number of mechanisms may be responsible for such effects. These include prevention of arrhythmias as well as lowering heart rate and blood pressure, decreasing platelet aggregation, and lowering triglyceride levels. The latter is accomplished by decreasing the production of hepatic triglycerides and increasing the clearance of plasma triglycerides (Harris et al., 2008). Fish oil is rich sources of the essential fatty acids eicosapentaenoic acid (EPA) and docosahexaenoic acid (DHA). Compared to saturated fats, PUFAs are more readily used for energy when initially ingested. The degree of unsaturation at a given carbon chain length was increasing the relative mobility of stored fat, making PUFAs more bioavailable (Aktay et al., 2000). In Isoproterenol (ISP) induced myocardial infarction rat model, fish oil oral supplementation showed cardio protective activity against ISP significant elevated serum ALT, AST and LDL by their significant reduction in a mechanism of antioxidant defense (Gopal et al., 2011). Farrah et al., (2018) found that the supporting the strong antioxidant and anti-inflammatory effects of fish oil supplementation. A large body of evidence suggests that limiting inflammation and cytokine production by dietary n-3 PUFA (EPA and DHA) represents one of the potential mechanisms by which fish oil is atheroprotective (Renier et al., 1993 and Blok et al., 1996). Recent study showed that fish oil daily supplementation led to significant improvement in cardiovascular parameters MDA, NO, GSH and TG levels as well as the lipid profile 
parameters in diabetics (Pingali et al., 2020).

\section{Conclusion}

The results of the present study suggest that the corn oil oral administration triggered risk factors for cardiovascular disease. While, fish oil and coconut oil administration showed protective activity against these factors with the superiority of fish oil over coconut oil.

\section{References}

Aktay, G., D. Deliorman, E. Ergun, F. Ergun, E. Yeşilada and C. Cevik, 2000. Hepatoprotective effects of Turkish folk remedies on experimental liver injury. Journal of Ethnopharmacology 73 (1-2):121-129.

Awabdy, D., L. J. Bryan-Lluka, and J. C. Wanstall, 2003. 5-Hydroxytryptamine and platelets: uptake and aggregation in hypoxic pulmonary hypertensive rats. European Journal of Pharmacology 459 (1):1-7.

Azeez, S., 2007. Fatty acid profile of coconut oil in relation to nut maturity and season in selected cultivars/hybrids. British Food Journal, 109(4): 272-279.

Baginski, E. S., E. Epstein, and B. Zak. 1972. The measurement of serum total phospholipids. Annals of Clinical \& Laboratory Science, 2 (4):255-267.

Banchroft, J.D., A. Stevens, and D.R. Turner, 1996. Theory and practice of histological techniques. Fourth Ed. Churchil Livingstone, New York, London, San Francisco, Tokyo. Pub. Date: January 1996; ISBN-13:9780443047602-Publisher: Elsevier Health Sciences, ISBN: 044304760X.

Banes, A. K. and S. W. Watts, 2003. Arterial expression of 5-HT 2B and 5-HT 1B receptors during development of DOCA-salt hypertension. BMC pharmacology, 3 (1):1-15.

Barrera-Arellano, D., A.P. Badan-Ribeiro, and S.O. Serna-Saldivar, 2019. Corn oil: composition, processing, and utilization. In Corn, 593-613: Elsevier.

Bian, K., M.F. Doursout and F. Murad. 2008. Vascular system: role of nitric oxide in cardiovascular diseases. The Journal of Clinical Hypertension, 10 (4):304-310.

Blok, W. L., M. B. Katan, and J. W. van der Meer, 1996. Modulation of inflammation and cytokine production by dietary (n-3) fatty acids. The Journal of Nutrition, 126 (6):1515-1533.

Burhani, M. D., and M. M. Rasenick. 2017. Fish oil and depression: the skinny on fats. Journal of Integrative Neuroscience, 16 (s1):S115-S124.

Carrillo, W., C. Carpio, D. Morales, E. Vilcacundo, M. Álvarez and M. Silva, 2017. Content of fatty acids in corn (Zea mays L.) oil from Ecuador. Asian Journal of Pharmaceutical and Clinical Research, 10:150-153.

Castro, V.M., C.C. Clements, S.N. Murphy, V.S. Gainer, M. Fava, J.B. Weilburg, J.L. Erb, S.E. Churchill, I.S. Kohane and D. V. Iosifescu, 2013. QT interval and antidepressant use: a cross sectional study of electronic health records. Bmj 346:f288.

Côté, F., C. Fligny, Y. Fromes, J. Mallet, and G. Vodjdani, 2004. Recent advances in understanding serotonin regulation of cardiovascular function. Trends in Molecular Medicine, 10 (5):232-238.

Dayton, S., S. Hashimoto, and M.L. Pearce, 1965. Influence of a diet high in unsaturated fat upon composition of arterial tissue and atheromata in man. Circulation, 32 (6):911-924.

Demaison, L. and D. Moreau, 2002. Dietary n-3 polyunsaturated fatty acids and coronary heart disease-related mortality: a possible mechanism of action. Cellular and Molecular Life Sciences CMLS, 59 (3):463-477.

Eid, R.A., M.A. Alkhateeb, S.M. Eleawa, M.S.A. Zaki, A.F. El-Kott, F. El-Sayed, H. Otifi, S. Alqahtani, Z.A. Asiri and H. Aldera, 2019. Fas/FasL-mediated cell death in rat's diabetic hearts involves activation of calcineurin/NFAT4 and is potentiated by a high-fat diet rich in corn oil. The Journal of Nutritional Biochemistry 68:79-90.

Eiselein, L., D.W. Wilson, M.W. Lamé, and J.C. Rutledge, 2007. Lipolysis products from triglyceride-rich lipoproteins increase endothelial permeability, perturb zonula occludens- 1 and F-actin, and induce apoptosis. American Journal of Physiology-Heart and Circulatory Physiology, 292 (6):H2745-H2753. 
El-Sayed, M.E.S.Y., R.M. Elsanhoty and M.F. Ramadan, 2014. Impact of dietary oils and fats on lipid peroxidation in liver and blood of albino rats. Asian Pacific Journal of Tropical Biomedicine, 4 (1):52-58.

Farrah, K., A. Farid and A. Mohammed, 2018. Antioxidant and anti-inflammatory effects of flaxseed oil and fish oil in fipronil induced oxidative stress in rats. Benha Veterinary Medical Journal, 35 (2):44-56.

Francis, G. A., 2000. High density lipoprotein oxidation: in vitro susceptibility and potential in vivo consequences. Biochimica et Biophysica Acta (BBA)-Molecular and Cell Biology of Lipids, 1483 (2):217-235.

Gershon, M., 1999. Roles played by 5-hydroxytryptamine in the physiology of the bowel. Alimentary Pharmacology \& Therapeutics, 13:15-30.

Gomaa, A. M. and E.A. Abd El-Aziz, 2016. Omega-3 fatty acids decreases oxidative stress, tumor necrosis factor-alpha, and interleukin-1 beta in hyperthyroidism-induced hepatic dysfunction rat model. Pathophysiology, 23(4):295-301.

Gopal, K. M., J. Mohan, M. Meganathan, P. Sasikala, N. Gowdhaman, K. Balamurugan, P. Nirmala, and A. S. Santhakumari, 2011. Effect of dietary fish oil (omega-3-fatty acid) against oxidative stress in isoproterenol induced myocardial injury in albino wistar rats. Global Journal of Pharmacology, 5(1):04-06.

Gumbiner, B., C.C. Low, and P.D. Reaven, 1998. Effects of a monounsaturated fatty acid-enriched hypocaloric diet on cardiovascular risk factors in obese patients with type 2 diabetes. Diabetes Care, 21(1):9-15.

Gylling, H. and T.A. Miettinen, 1999. Cholesterol reduction by different plant stanol mixtures and with variable fat intake. Metabolism, 48(5):575-580.

Hagen, T. M., and D. P. Jones. 1989. Role of glutathione transport in extra hepatic detoxication. In Glutathione Centennial, 423-433: Elsevier.

Hajar, R., 2017. Risk factors for coronary artery disease: historical perspectives. Heart views: the official journal of the Gulf Heart Association, 18 (3): 109.

Hallikainen, M. A., E. Sarkkinen, H. Gylling, A. Erkkilä, and M. Uusitupa, 2000. Comparison of the effects of plant sterol ester and plant stanol ester-enriched margarines in lowering serum cholesterol concentrations in hypercholesterolaemic subjects on a low-fat diet. European Journal of Clinical Nutrition, 54(9):715-725.

Harris, W.S., 1989. Fish oils and plasma lipid and lipoprotein metabolism in humans: a critical review. Journal of Lipid Research, 30 (6):785-807.

Harris, W.S., M. Miller, A.P. Tighe, M.H. Davidson, and E.J. Schaefer, 2008. Omega-3 fatty acids and coronary heart disease risk: clinical and mechanistic perspectives. Atherosclerosis, 197 (1):12-24

He, J., L. G. Ogden, L. A. Bazzano, S. Vupputuri, C. Loria, and P.K. Whelton, 2001. Risk factors for congestive heart failure in US men and women: NHANES I epidemiologic follow-up study. Archives of Internal Medicine, 161 (7):996-1002.

Hu, F.B., J.E. Manson and W.C. Willett, 2001. Types of dietary fat and risk of coronary heart disease: a critical review. Journal of the American College of Nutrition, 20 (1):5-19.

Jacobs, B.L., and E.C. Azmitia, 1992. Structure and function of the brain serotonin system. Physiological Reviews, 72 (1):165-229.

Jayatilleke, E., and S. Shaw. 1993. A high-performance liquid chromatographic assay for reduced and oxidized glutathione in biological samples. Analytical Biochemistry, 214(2):452-457.

Jira, W., G. Spiteller, W. Carson, and A. Schramm. 1998. Strong increase in hydroxy fatty acids derived from linoleic acid in human low density lipoproteins of atherosclerotic patients. Chemistry and Physics of Lipids, 91(1):1-11.

Kannel, W.B., and D.L. McGee. 1979. Diabetes and cardiovascular risk factors: the Framingham study. Circulation, 59 (1):8-13.

Karatepe, M., 2004. Simultaneous determination of ascorbic acid and free malondialdehyde in human serum by HPLC-UV. Lc Gc North America, 22(4):362-365. 
Kris-Etherton, P.M., K.D. Hecker, and A.E. Binkoski, 2004. Polyunsaturated fatty acids and cardiovascular health. Nutrition Reviews, 62(11):414-426.

Leong, X.F., A. Aishah, U.N. Aini, S. Das, and K. Jaarin, 2008. Heated palm oil causes rise in blood pressure and cardiac changes in heart muscle in experimental rats. Archives of Medical Research, 39 (6):567-572.

Li, L., S. Morimoto, S. Take, D.Y. Zhan, C.K. Du, Y.Y. Wang, X.L. Fan, T. Yoshihara, F. TakahashiYanaga and T. Katafuchi, 2012. Role of brain serotonin dysfunction in the pathophysiology of congestive heart failure. Journal of Molecular and Cellular Cardiology, 53 (6):760-767.

Lichtenstein, A.H., L.J. Appel, M. Brands, M. Carnethon, S. Daniels, H.A. Franch, B. Franklin, P. Kris-Etherton, W.S. Harris and B. Howard, 2006. Diet and lifestyle recommendations revision 2006: a scientific statement from the American Heart Association Nutrition Committee. Circulation, 114 (1):82-96.

Mason, P., S. Porter, S. Berry, P. Stillman, C. Steele, A. Kirby, B. Griffin, and A. Minihane, 2009. Saturated fatty acid consumption: outlining the scale of the problem and assessing the solutions. Nutrition Bulletin, 34 (1):74-84.

Masters, R.K., E.N. Reither, D.A. Powers, Y.C. Yang, A.E. Burger, and B.G. Link, 2013. The impact of obesity on US mortality levels: the importance of age and cohort factors in population estimates. American Journal of Public Health, 103(10):1895-1901.

Mensink, R. P., and M. B. Katan. 1992. Effect of dietary fatty acids on serum lipids and lipoproteins. A meta-analysis of 27 trials. Arteriosclerosis and thrombosis: A Journal of Vascular Biology, 12(8):911-919.

Narayanankutty, A., S. P. Illam, and A. C. Raghavamenon. 2018. Health impacts of different edible oils prepared from coconut (Cocos nucifera): A comprehensive review. Trends in Food Science \& Technology, 80:1-7.

Nebigil, C.G., P. Hickel, N. Messaddeq, J.L. Vonesch, M.P. Douchet, L. Monassier, K. György, R. Matz, R. Andriantsitohaina, and P. Manivet, 2001. Ablation of serotonin 5-HT2B receptors in mice leads to abnormal cardiac structure and function. Circulation, 103(24):2973-2979.

Nicolosi, R.J., T.A. Wilson, G. Handelman, T. Foxall, J. F. Keaney Jr and J.A. Vita. 2002. Decreased aortic early atherosclerosis in hypercholesterolemic hamsters fed oleic acid-rich TriSun oil compared to linoleic acid-rich sunflower oil. The Journal of Nutritional Biochemistry, 13 (7):392-402.

O'Connor, S. E., A. Grosset, C. D. La Rochelle, E. Gautier, J.-P. Bidouard, P. Robineau, D. Caille, and P. Janiak. 2001. Cardiovascular effects of SL65. 0472, a 5-HT receptor antagonist. European Journal of Pharmacology, 414 (2-3):259-269.

O'Donnell, C. J., and R. Elosua. 2008. Cardiovascular risk factors. Insights from framingham heart study. Revista Española de Cardiología (English Edition) 61 (3):299-310.

Pagel, P., J. Blome, and H.U. Wolf, 2000. High-performance liquid chromatographic separation and measurement of various biogenic compounds possibly involved in the pathomechanism of Parkinson's disease. Journal of Chromatography B: Biomedical Sciences and Applications, 746 (2):297-304.

Papadoyannis, I., V. Samanidou, and C.C. Nitsos, 1999. Simultaneous determination of nitrite and nitrate in drinking water and human serum by high performance anion-exchange chromatography and UV detection. Journal of Liquid Chromatography \& Related Technologies, 22 (13):2023-2041.

Pingali, U., C. Nutalapati, and V.S. Illendulla, 2020. Evaluation of the Effect of Fish Oil Alone and in Combination with a Proprietary Chromium Complex on Endothelial Dysfunction, Systemic Inflammation and Lipid Profile in Type 2 Diabetes Mellitus-A Randomized, Double-Blind, Placebo-Controlled Clinical Study. Diabetes, Metabolic Syndrome and Obesity: Targets and Therapy, 13:31.

Plat, J., and R.P. Mensink. 2000. Vegetable oil based versus wood based stanol ester mixtures: effects on serum lipids and hemostatic factors in non-hypercholesterolemic subjects. Atherosclerosis, 148 (1):101-112.

Prasad, K., 1997. Dietary flax seed in prevention of hypercholesterolemic atherosclerosis. Atherosclerosis, 132 (1):69-76. 
Prasad, K., and J. Kalra. 1993. Oxygen free radicals and hypercholesterolemic atherosclerosis: effect of vitamin E. American Heart Journal, 125 (4):958-973.

Raddino, R., G. Caretta, M. Teli, I. Bonadei, D. Robba, G. Zanini, A. Madureri, S. Nodari, and L. Dei Cas. 2007. Nitric oxide and cardiovascular risk factors. Heart International, 3(12):1826186807003001-203.

Reaven, P., S. Parthasarathy, B. J. Grasse, E. Miller, D. Steinberg, and J. L. Witztum, 1993. Effects of oleate-rich and linoleate-rich diets on the susceptibility of low density lipoprotein to oxidative modification in mildly hypercholesterolemic subjects. The Journal of Clinical Investigation, 91 (2):668-676.

Reeves, P.G., F.H. Nielsen, and G.C. Fahey Jr., 1993. AIN-93 purified diets for laboratory rodents: final report of the American Institute of Nutrition ad hoc writing committee on the reformulation of the AIN-76A rodent diet: Oxford University Press.

Renier, G., E. Skamene, J. DeSanctis, and D. Radzioch, 1993. Dietary n-3 polyunsaturated fatty acids prevent the development of atherosclerotic lesions in mice. Modulation of macrophage secretory activities. Arteriosclerosis and Thrombosis: A Journal of Vascular Biology, 13 (10):1515-1524.

Resende, N.M., H.R. Felix, M.R. Sore, A. Mmneto, K.E. Campos and G.T. Volpato, 2016. The effects of coconut oil supplementation on the body composition and lipid profile of rats submitted to physical exercise. Anais da Academia Brasileira de Ciências, 88 (2):933-940.

Rohman, A., Irnawati, Y. Erwanto, E. Lukitaningsih, M. Rafi, N. A. Fadzilah, A. Windarsih, A. Sulaiman, and Z. Zakaria. 2019. Virgin coconut oil: Extraction, physicochemical properties, biological activities and its authentication analysis. Food Reviews International, 1-21.

Santos, H. O., S. Howell, C. P. Earnest, and F. J. Teixeira. 2019. Coconut oil intake and its effects on the cardiometabolic profile-A structured literature review. Progress in Cardiovascular Diseases 62 (5): 436-443.

Savva, S. C., and A. Kafatos, 2015. Vegetable Oils: Dietary Importance. In Encyclopedia of Food and Health (pp. 365-372). Elsevier Inc.

Shao, B., M.N. Oda, T. Vaisar, J.F. Oram, and J.W. Heinecke, 2006. Pathways for oxidation of highdensity lipoprotein in human cardiovascular disease. Current opinion in molecular therapeutics, 8 (3):198-205.

Shimizu, H., Y. Kiyohara, I. Kato, T. Kitazono, Y. Tanizaki, M. Kubo, H. Ueno, S. Ibayashi, M. Fujishima, and M. Iida. 2004. Relationship between plasma glutathione levels and cardiovascular disease in a defined population: the Hisayama study. Stroke, 35 (9):2072-2077.

Silaste, M.L., M. Rantala, G. Alfthan, A. Aro, J. L. Witztum, Y. A. Kesäniemi and S. Hörkkö. 2004. Changes in dietary fat intake alter plasma levels of oxidized low-density lipoprotein and lipoprotein (a). Arteriosclerosis, Thrombosis, and Vascular Biology, 24(3):498-503.

Stamler, J. S., and A. Slivka. 1996. Biological chemistry of thiols in the vasculature and in vascularrelated disease. Nutrition Reviews, 54 (1):1-30.

Teerlink, T., M. Hennekes, J. Bussemaker and J. Groeneveld. 1993. Simultaneous determination of creatine compounds and adenine nucleotides in myocardial tissue by high-performance liquid chromatography. Analytical Biochemistry 214 (1):278-283.

Vanhoutte, P. M. 1987. Cardiovascular effects of serotonin. Journal of cardiovascular pharmacology 10:S8-S11.

Voelker, R., 2019. Oleic acid can make heart claim without hard evidence. Jama, 321 (1):23-23.

Wang, L., R. Gill, T.L. Pedersen, L.J. Higgins, J.W. Newman and J.C. Rutledge, 2009. Triglyceriderich lipoprotein lipolysis releases neutral and oxidized FFAs that induce endothelial cell inflammation. Journal of Lipid Research, 50 (2):204-213.

Wooten, J.S., T.N. Nick, A. Seija, K.E. Poole, and K.B. Stout, 2016. High-fructose intake impairs the hepatic hypolipidemic effects of a high-fat fish-oil diet in C57BL/6 mice. Journal of Clinical and Experimental Hepatology, 6(4):265-274.

Yamagishi, K., H. Iso, H. Yatsuya, N. Tanabe, C. Date, S. Kikuchi, A. Yamamoto, Y. Inaba, and A. Tamakoshi, 2010. Dietary intake of saturated fatty acids and mortality from cardiovascular disease in Japanese: the Japan Collaborative Cohort Study for Evaluation of Cancer Risk (JACC) Study. Am J Clin Nutr., 92(4):759-765. 
Yildiz, O., J. Smith, and R. Purdy. 1998. Serotonin and vasoconstrictor synergism. Life Sciences, 62 (19):1723-1732.

Young, I., C. McFarlane, and J. McEneny, 2003. Oxidative modification of triacylglycerol-rich lipoproteins: Portland Press Ltd. 